\title{
Self-similar critical geometries at horizon intersections and mergers
}

\author{
Roberto Emparan $^{a, b}$, Nidal Haddad Ha $^{a}$ \\ ${ }^{a}$ Departament de Física Fonamental and \\ Institut de Ciències del Cosmos, Universitat de Barcelona, \\ Martí i Franquès 1, E-08028 Barcelona, Spain \\ ${ }^{b}$ Institució Catalana de Recerca i Estudis Avançats (ICREA) \\ Passeig Lluís Companys 23, E-08010 Barcelona, Spain \\ emparan@ub.edu, nidal@ffn.ub.es
}

\begin{abstract}
We study topology-changing transitions in the space of higher-dimensional black hole solutions. Kol has proposed that these are conifold-type transitions controlled by self-similar double-cone geometries. We present an exact example of this phenomenon in the intersection between a black hole horizon and a cosmological deSitter horizon in $D \geq 6$. We also describe local models for the critical geometries that control many transitions in the phase space of higher-dimensional black holes, such as the pinchdown of a topologically spherical black hole to a black ring or to a black $p$-sphere, or the merger between black holes and black rings in black Saturns or di-rings in $D \geq 6$.
\end{abstract}




\section{Introduction}

Black holes in higher dimensions exhibit a pattern of phases much more intricate than the simple situation that uniqueness theorems impose in four dimensions. Unraveling the structure of this phase space is a problem in which, despite the steady progress in recent years, there remain important open issues. In particular, the topology-changing transitions in the space of solutions at which horizons split or merge involve all the nonlinearity of Einstein's theory and lie far from the regimes where analytic perturbative techniques of the type developed in [1, 2] would apply. Numerical methods, while very valuable, face the problem that the geometries that effect the change in topology involve curvature singularities.

The most studied example of this phenomenon 1 is the black hole-black string transition in Kaluza-Klein compactified spacetimes [3]. The uniform string phase branches into a non-uniform black string phase [4] whose non-uniformity grows until a cycle along the horizon of the string pinches down to zero size. The same pinched-off phase can be approached from black holes localized in the KK circle, where the size of the black hole grows until the two opposite poles of the horizon come into contact with each other along the circle [5] (see ref. [6] for the state of the art in solutions on both sides of the transition). Early in these studies, ref. [7] emphasized that the pinched-off solution plays the role of a critical point in phase space and argued that some of its properties, in particular the local geometry near the singular pinch-off region, should be determined by general symmetry considerations. Specifically, it was proposed, using an argument that we summarize in fig. 1, that this geometry (after Wick-rotation to Euclidean time) is locally modelled by a self-similar cone over $S^{2} \times S^{D-3}$. The transition between phases is analogous to the 'conifold transition', where the critical cone geometry can be smoothed in two ways, each one leading to one of the phases at each side of the transition.

Ref. [7] could present in exact form only the local conical geometry asymptotically close to the pinch-off point. The details of how, and even whether, this conical region extends to a full critical solution in the KK circle remained open. Moreover, the nonsingular geometries that approach the critical solution are only known perturbatively or numerically (see [8, 9] and other works cited above). The aim of this paper is to, first, present an exact example of a horizon-merger transition that provides strong analytic evidence for the conifold-type picture, and second, to argue for the universality of this picture among wide classes of topology-changing transitions involving higherdimensional black hole phases.

\footnotetext{
${ }^{1}$ In this paper we are concerned only with evolution in the phase space of stationary black hole solutions, not with time evolution in dynamical mergers of horizons.
} 


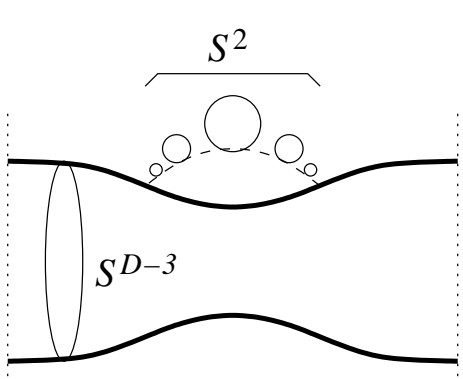

(a)

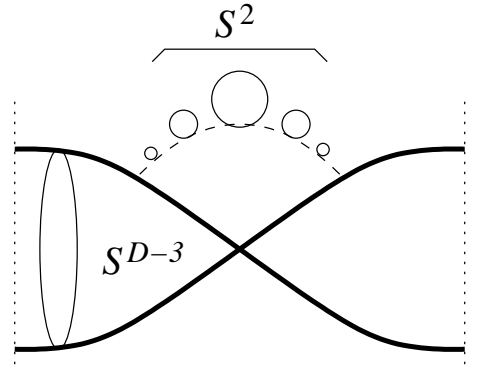

(b)

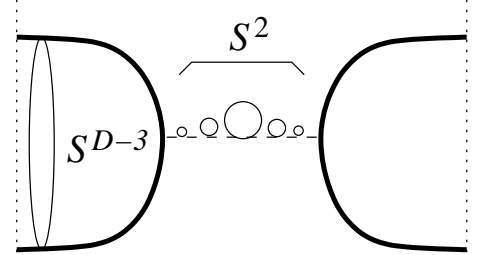

(c)

Figure 1: Black hole/black string transition in a Kaluza-Klein circle (the circle runs along the horizontal axis), following [7]. The circle fibered over the dashed segments is the Euclidean time circle, which shrinks to zero at the horizon: this fiber bundle describes a $S^{2}$. We also mark a cycle $S^{D-3}$ on the horizon. In the black string phase (a) the $S^{2}$ is contractible, the $S^{D-3}$ is not, while in the black hole phase (c) the $S^{D-3}$ is contractible, the $S^{2}$ is not. The transition between the two phases is of conifold type, with the critical geometry (b) becoming, near the pinch-off point, a cone over $S^{2} \times S^{D-3}$.

To this end, in section 2 we present a complete, exact family of geometries that approach a merger of horizons, and we give explicitly the critical solution at the merger. The system describes the meeting of the horizon of a black hole with a cosmological deSitter horizon in any $D \geq 6$, with the black hole sufficiently distorted away from spherical symmetry that it intersects the deSitter horizon along a circle, and not on all points on the horizon. This study confirms that the critical solution near the pinch-off is locally a self-similar cone with the geometry anticipated in [7]. Section 3 is an attempt to find the geometry after the horizons have merged into a single one. Unfortunately, the result is only partially successful since the solution presents some pathologies.

In section 4 we describe the critical geometries at the merger point in other important instances - here we do not have a description of the approach to the merger transition, but we can always identify the local model for the critical geometries. Refs. [10, 11] (following [12]) have proposed the following picture for a specific class of topology-changing transitions: a black ring of horizon topology $S^{1} \times S^{D-3}$ in $D \geq 6$ becomes, as its spin decreases, fat enough that its hole closes up. Coming from the other side of the transition, the same phase is reached when a pinched rotating black hole pinches off to a singularity at its axis of rotation. In this paper we describe the local conical geometry that controls this transition. We also extend the analysis to similar transitions that involve black holes with horizon topology $S^{p} \times S^{D-p-2}$, with $p \geq 1$ [1, 13]. We find the critical conical geometries that appear when the round $S^{p}$ closes off and the critical solution connects to a black hole of spherical topology. Other 

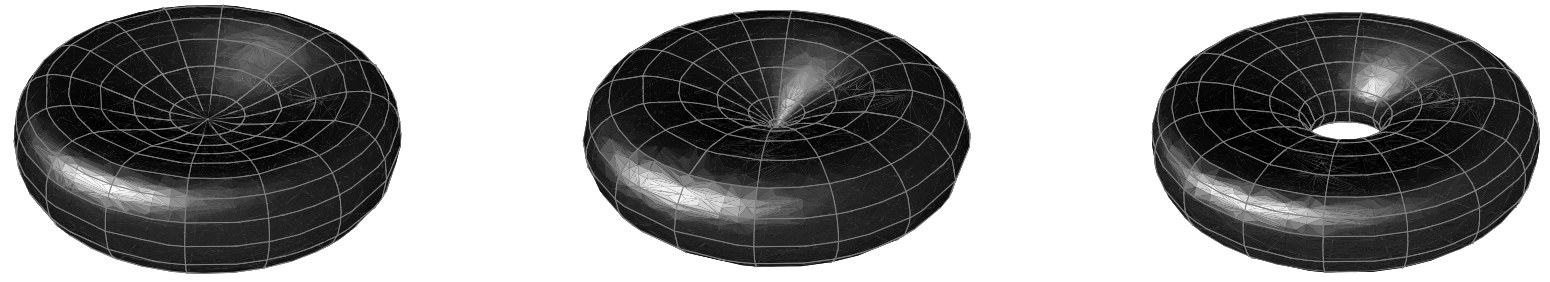

Figure 2: Black ring pinch in $D \geq 6$. The pictures are only illustrative of expected solutions that are yet to be constructed. In sec. 4 we describe the critical geometry near the self-similar pinch-off point.

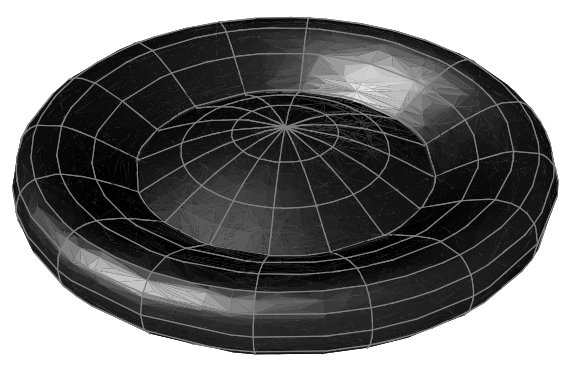

Figure 3: Circular pinch in the transition involving a black Saturn in $D \geq 6$.

conical geometries, for instance those that appear at the merger of a black ring and a black hole, or two black rings, are easily obtained too.

An interesting, perhaps unexpected, consequence of our analysis is that the critical conical geometries appear not only when horizons merge, but more generally when they just intersect. To understand the distinction, note that if there is an actual transition in which two separate horizons merge to form one horizon, the surface gravities (i.e., temperatures) of the two horizons must approach the same value at the critical solution. However, we find exact critical solutions in which the two horizons have different surface gravities. These horizons can approach and touch each other locally, i.e., intersect, over a singularity, but they cannot merge to form a single, connected horizon over which the temperature must be uniform. Such intersection geometries correspond to endpoints of trajectories in the space of solutions, and not to topology-changing transitions. We find that they also take the form of self-similar cones, but their base is not a homogeneous space (a direct product of round spheres) but an inhomogeneous one (a warped product). 
Caveat emptor, evolution in the space of black hole solutions, as studied in this paper, occurs along geometries that can be quite different than in dynamical evolution in time, as the example of the black string/black hole transition shows [14]. Presumably this is also the case in the instances we discuss here.

\section{Intersection of black hole and deSitter horizons}

The so-called $D$-dimensional Kerr-deSitter solution for a rotating black hole in deSitter space with a single rotation, as found in [15] but with a shift $\phi \rightarrow \phi+a t / L^{2}$, is

$$
\begin{aligned}
d s^{2}= & -\frac{\Delta_{r}}{\rho^{2} \Xi^{2}}\left(\Delta_{\theta} d t-a \sin ^{2} \theta d \phi\right)^{2}+\rho^{2}\left(\frac{d r^{2}}{\Delta_{r}}+\frac{d \theta^{2}}{\Delta_{\theta}}\right) \\
& +\frac{\Delta_{\theta} \sin ^{2} \theta}{\rho^{2} \Xi^{2}}\left(\left(r^{2}+a^{2}\right) d \phi-a\left(1-\frac{r^{2}}{L^{2}}\right) d t\right)^{2}+r^{2} \cos ^{2} \theta d \Omega_{(D-4)}^{2}
\end{aligned}
$$

with

$$
\begin{aligned}
\rho^{2} & =r^{2}+a^{2} \cos ^{2} \theta, \\
\Delta_{r} & =\left(r^{2}+a^{2}\right)\left(1-\frac{r^{2}}{L^{2}}\right)-\frac{2 M}{r^{D-5}}, \\
\Delta_{\theta} & =1+\frac{a^{2}}{L^{2}} \cos ^{2} \theta, \\
\Xi & =1+\frac{a^{2}}{L^{2}},
\end{aligned}
$$

and

$$
0 \leq \theta \leq \frac{\pi}{2}, \quad 0 \leq \phi \leq 2 \pi
$$

The metric satisfies $R_{\mu \nu}=(D-1) L^{-2} g_{\mu \nu}$. When $M=0$ this is deSitter spacetime in 'ellipsoidal coordinates'. When $M$ is non-zero and positive, there is a range of values of $M$ and $a$ for which the function $\Delta_{r}$ has two real positive roots that correspond to the black hole and cosmological horizons. With non-zero rotation $a \neq 0$, both horizons are distorted away from spherical symmetry. For reasons that will become apparent, we only consider $D \geq 6$.

\subsection{Horizon intersection}

We want to take a limit in which the black hole grows and its horizon touches the cosmological horizon, in such a way that this occurs not uniformly over all of the horizon, but only along the 'equator' at $\theta=\pi / 2$, as illustrated in fig. 4 . There is an intuitive reason why this should be possible in $D \geq 6$ : in these dimensions, Myers-Perry 


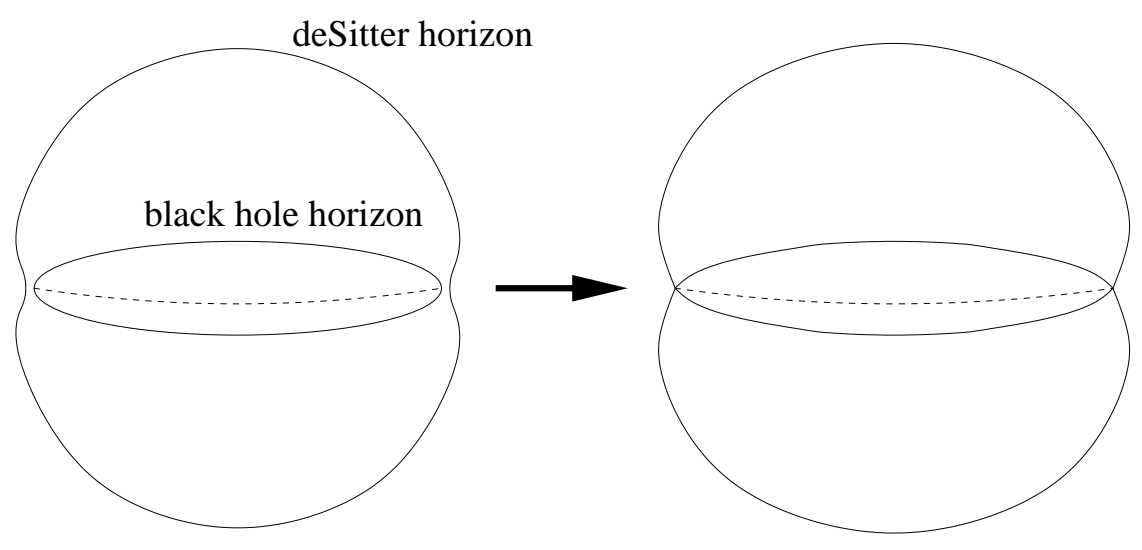

Figure 4: Sketch of black hole-deSitter horizons in the approach to the solution in which the black hole touches the deSitter (cosmological) horizon along its equator. Only in $D \geq 6$ does the black hole admit a large enough distortion away from spherical symmetry to allow this type of configuration.

black holes rotating along one plane can become very flat and thin, effectively like disks of a black membrane [12]. The blackfold approach of [1, 2] allows to construct a static configuration in which this disk extends along a plane and touches the deSitter horizon. This blackfold construction is easy to perform and gives an approximate solution for the intersecting-horizon configuration. However, we will not give its details since we can obtain the complete exact solution that it is an approximation to.

The appropriate limit of (2.1) is

$$
a, M \rightarrow \infty
$$

keeping fixed

$$
\mu=\frac{2 M}{a^{2}}
$$

Even if we are taking the rotation parameter $a$ to be very large, this does not mean that the black hole rotates very rapidly relative to the cosmological horizon. By taking $M$ large we are also making the black hole size grow. The relative drag between the two horizons increases and as a consequence they approach corotation, with the relative angular velocity decreasing. Eventually, when the black hole touches the deSitter horizon, the configuration becomes manifestly static with our choice of coordinates. However, as we shall see presently, since the distortion away from spherical symmetry remains in the limit, the horizons touch only along the equator. 
Taking the above limit in (2.1), we find

$$
\begin{aligned}
d s^{2}= & L^{2}\left(d \theta^{2}+\sin ^{2} \theta d \phi^{2}\right) \\
& +\cos ^{2} \theta\left(-\left(1-\frac{\mu}{r^{D-5}}-\frac{r^{2}}{L^{2}}\right) d t^{2}+\frac{d r^{2}}{1-\frac{\mu}{r^{D-5}}-\frac{r^{2}}{L^{2}}}+r^{2} d \Omega_{(D-4)}^{2}\right) \\
= & L^{2}\left(d \theta^{2}+\sin ^{2} \theta d \phi^{2}\right)+\cos ^{2} \theta d s^{2}\left(\text { Schw }-\mathrm{dS} S_{D-2}\right),
\end{aligned}
$$

where Schw-dS ${ }_{D-2}$ denotes the Schwarzschild-deSitter geometry in $D-2$ dimensions. When $\mu=0$ this factor becomes $(D-2)$-dimensional deSitter $\left(\mathrm{dS}_{D-2}\right)$ and the whole geometry is $\mathrm{dS}_{D}$ spacetime of radius $L$. The singularity at $\theta=\pi / 2$ in this metric is just a coordinate artifact. The cosmological horizon at $r=L$ has the usual geometry of a round $S^{D-2}$.

Our actual interest is in the solutions with $\mu \neq 0$. Then the singularity at $\theta=\pi / 2$ is a true one where the curvature diverges. Setting $\theta-\pi / 2=z / L$, then near $z=0$ the geometry asymptotically becomes

$$
d s^{2} \rightarrow d z^{2}+L^{2} d \phi^{2}+\frac{z^{2}}{L^{2}} d s^{2}\left(\mathrm{Schw}-\mathrm{dS}{ }_{D-2}\right)
$$

This has the form of a cone over Schw- $\mathrm{dS}_{D-2}$, spread along the circle generated by $\phi$.

Before we took the limit, the solution (2.1) in the parameter range of interest described two separate horizons, black hole and cosmological. In the limiting solution, these are still present. The equation

$$
\frac{r^{2}}{L^{2}}+\frac{\mu}{r^{D-5}}=1
$$

with

$$
0<\frac{\mu}{L^{D-5}}<\frac{2}{D-5}\left(\frac{D-5}{D-3}\right)^{\frac{D-3}{2}}
$$

has two real positive roots for $r$, for the black hole and cosmological horizons in the Schw-dS $\mathrm{S}_{D-2}$ sub-spacetime. These are the limits of the black hole and cosmological horizons of the original solution (2.1) in $D$ dimensions. In (2.9) these two horizons come to touch each other along the circle $\theta=\pi / 2$.

The continuously self-similar structure of a cone around this intersection point is apparent in (2.10). If we analytically continue to Euclidean time $\tau$, then the $(\tau, r)$ part of the Schw- $\mathrm{dS}_{D-2}$ geometry describes a two-sphere, generically with a conical defect (of codimension 1) at either the cosmological or black hole horizons since their temperatures are not the same for generic values of $\mu / L^{D-5}$. Except for this defect the Euclidean geometry of Schw-dS ${ }_{D-2}$ is a warped product of $S_{(\tau, r)}^{2}$ and $S^{D-4}$. So the Euclidean continuation of (2.10) is a cone over this warped $S^{2} \times S^{D-4}$, times the $\phi$ 
circle. This cone is essentially the local model for the critical geometry that [7] had proposed.

Note, however, that what we have found is a more general cone than in [7]. In (2.9) the temperatures of the black hole and of the cosmological horizons need not be the same. For instance, in configurations where the black hole has the shape of a very thin pancake, which occur when $\mu \ll L^{D-5}$, the black hole temperature is clearly much higher than the temperature of the cosmological horizon 2

When their temperatures are different, the two horizons can approach each other and intersect, but not evolve beyond the intersection to merge and form a single, connected horizon. Since the temperature over the latter must be uniform, a merger requires that the temperatures of the two horizons approach the same value at the intersection. Ref. [7] focused on merger transitions, but we have seen that the appearance of self-similar conical geometries is a more general feature of intersections of horizons, even when they cannot merge. For the Lorentzian solutions the difference in temperatures does not imply any pathology, so it is natural to consider these configurations as well.

The parameter $\mu$ in the critical geometry (2.9) can be adjusted so that the two horizons have the same surface gravity. In fact it is possible to consider not only this solution, but an entire subfamily of solutions of (2.1) in which the two separate horizons, black hole and cosmological, have the same surface gravity even away from the critical merger geometry. This subfamily of solutions describes a rotating black hole with the same temperature as the cosmological horizon, i.e., a 'rotating Nariai' solution, and is of some interest in itself, so we describe it next.

\subsection{Isothermal solutions}

The limit of the solutions (2.1) where the two separate horizons have equal temperatures, or surface gravities, is defined in appendix A. The mass and the radial coordinate are fixed to values $M=M_{0}$ and $r=r_{0}$ that depend on the rotation parameter $a$. Using for simplicity units where $L=1$, the metric that results is

$$
\begin{aligned}
d s^{2}= & C \rho_{0}^{2}\left(-\sin ^{2} \chi d \tilde{t}^{2}+d \chi^{2}\right)+\frac{\rho_{0}^{2}}{\Delta_{\theta}} d \theta^{2} \\
& +\frac{\Delta_{\theta} \sin ^{2} \theta}{\rho_{0}^{2} \Xi^{2}}\left(\left(r_{0}^{2}+a^{2}\right) d \tilde{\phi}-2 C r_{0} a \Xi \cos \chi d \tilde{t}\right)^{2}+r_{0}^{2} \cos ^{2} \theta d \Omega_{(D-4)}^{2} .
\end{aligned}
$$

\footnotetext{
${ }^{2}$ The blackfold method reproduces these configurations to leading order in $\mu / L^{D-5} \ll 1$. In the exact solutions one can take a limit to focus on the region very close to the axis $\theta=0$ and recover the geometry of a black 2-brane of thickness much smaller than $L$.
} 
where

$$
\rho_{0}^{2}=r_{0}^{2}+a^{2} \cos ^{2} \theta,
$$

and $\Delta_{\theta}, \Xi$ as in (2.4), (2.5) (with $L=1$ ) and

$$
0 \leq \chi \leq \pi, \quad 0 \leq \theta \leq \frac{\pi}{2}, \quad 0 \leq \tilde{\phi} \leq 2 \pi .
$$

The constants $r_{0}$ and $C$ are determined in terms of $a$ by

$$
a^{2}=r_{0}^{2} \frac{D-3-(D-1) r_{0}^{2}}{(D-3) r_{0}^{2}-(D-5)}
$$

and

$$
C=\frac{(D-3) r_{0}^{2}-(D-5)}{(D-1)(D-3) r_{0}^{4}-2(D-5)(D-1) r_{0}^{2}+(D-5)(D-3)} .
$$

Actually, we could take the only parameter in the solution to be $r_{0}$ instead of $a$, since they are related in one-to-one manner in the range of interest, which is

$$
0 \leq a \leq \infty, \quad \frac{D-5}{D-3} \leq r_{0}^{2} \leq \frac{D-3}{D-1}
$$

(note this implies $r_{0}^{2}<1$ ).

In going from (2.1) to (2.13), the cohomogeneity of the geometry has been reduced from two to one, the only non-trivial dependence being now on $\theta$. It is manifest that the surface gravities at the two horizons, at $\chi=0$ and $\chi=\pi$, are equal. However, there is a relative angular velocity between them,

$$
\Omega_{r e l}=\frac{4 C r_{0} a \Xi}{r_{0}^{2}+a^{2}} .
$$

For a merger of the two horizons to be possible, this relative motion between them must disappear at the critical solution.

In the conventional static limit $a \rightarrow 0$, where $r_{0}^{2} \rightarrow(D-3) /(D-1)$, eq. (2.13) becomes the Nariai limit of Schw- $\mathrm{dS}_{D}$, namely the direct product geometry

$$
d s^{2} \stackrel{a \rightarrow 0}{\longrightarrow} \frac{1}{D-1}\left(-\sin ^{2} \chi d \tilde{t}^{2}+d \chi^{2}\right)+\frac{D-3}{D-1} d \Omega_{(D-2)}^{2}
$$

which Wick-rotates to $S^{2} \times S^{D-2}$. In this solution, and in all the solutions with finite $a$, the two horizons remain separate.

The limit that we are interested in, where the two horizons touch at the equator $\theta=\pi / 2$, lies at $a \rightarrow \infty$, with $r_{0}^{2} \rightarrow(D-5) /(D-3)$. Then $\Omega_{r e l} \rightarrow 0$ (as we had already observed in the previous section) and

$$
d s^{2} \stackrel{a \rightarrow \infty}{\longrightarrow} \frac{1}{D-3} \cos ^{2} \theta\left(-\sin ^{2} \chi d \tilde{t}^{2}+d \chi^{2}\right)+d \theta^{2}+\sin ^{2} \theta d \tilde{\phi}^{2}+\frac{D-5}{D-3} \cos ^{2} \theta d \Omega_{(D-2)}^{2} .
$$


This is indeed the same geometry that results if we take the Nariai limit of the Schw$\mathrm{dS}_{D-2}$ geometry inside (2.9). We have obtained it here along a particular one-parameter subfamily of the solutions (2.1).

In the region close to the intersection of horizons, with small $\theta-\pi / 2=z / L$, the geometry (2.21) becomes, after rotation to Euclidean time (and restoring $L$ ),

$$
d s^{2} \rightarrow d z^{2}+L^{2} d \tilde{\phi}^{2}+\frac{z^{2}}{D-3}\left(d \Omega_{(2)}^{2}+(D-5) d \Omega_{(D-4)}^{2}\right)
$$

which is exactly the kind of double-cone geometry predicted by the arguments in [7].

We have studied the solutions in which the initial black hole rotates along a single plane, but it is straightforward to extend this to the general solutions with rotation in an arbitrary number of planes and then obtain intersections along odd-spheres instead of circles. Appendix B explains this construction.

\section{Merged solution}

It is natural now to look for an exact solution for the geometry after the two horizons have merged into a single one. Here we describe our attempt at finding this solution. In contrast to the pre-merger solution of the previous section, the merged solution that we find is not entirely satisfactory - its Lorentzian section is complex, and it has a naked singularity. It is unclear to us whether this is a deficiency somehow intrinsic to the (cosmological) set up that we are considering, or whether there is another solution for the merged configuration. Readers not interested in the details can safely jump to section 4 ,

One might expect, by analyticity in the space of solutions, that the solution after the black hole and cosmological horizons have merged should be in the same family as (2.13), but in a different parameter range than before the merger - i.e., in the merged solution the parameter $a$ extends beyond the range we have been considering in sec. 2.2. Actually, we will consider not only (2.13), or the initial Kerr-dS solution (2.1), but the Kerr-NUT-dS family. This is the largest known family of solutions that appear appropriate for this task. As in section 2, away from the critical solution we consider the Lorentzian section of the geometries.

It is useful to first identify the main properties that the solution must possess. First, the family of solutions should obviously have a limit to the critical geometry (2.21).

Second, from the general picture of the conifold-type transition, in the merged solution there should be a 'Lorentzian two-sphere' (like the one that $\tilde{t}$ and $\chi$ describe in (2.21) ) that is contractible to zero, and a $S^{D-4}$ that is not. Observe that this, and 


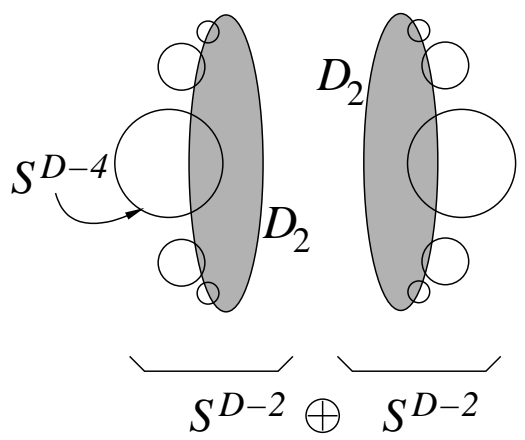

(a)

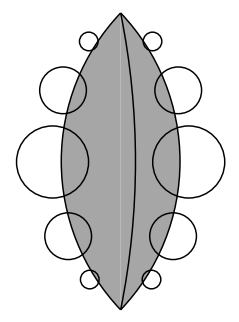

(b)

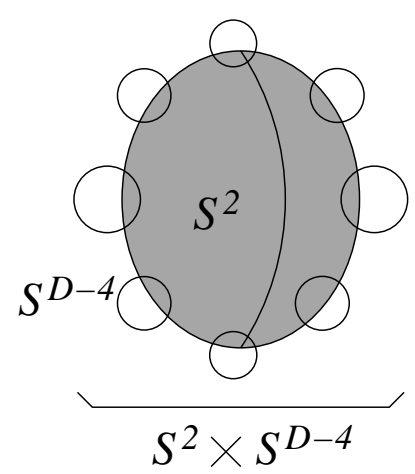

(c)

Figure 5: Horizon geometry in (a) the black hole-deSitter phase; (b) critical solution; (c) merged solution.

the first condition, rule out the simple possibility that when the two horizons merge we recover $\mathrm{dS}_{D}$.

Finally, we can easily determine the topology of the spatial sections of the horizon. As illustrated in figure 5, before the merger the horizon is the sum of two $S^{D-2}$. Each of these spheres can be viewed as the result of fibering the (topological) disk $D_{2}$, parametrized by $(\theta, \phi)$, with spheres $S^{D-4}$ whose size goes to zero at the boundary of the disk at $\theta=\pi / 2$. In the critical geometry, the two disks meet at their edges: they form a topological $S^{2}$. The spheres $S^{D-4}$ fiber over this $S^{2}$, with their sizes shrinking to zero at the circle where the two disks meet: this is the horizon of the critical solution. When the two horizons merge to form a single one, the $S^{D-4}$ do not shrink to zero anywhere, so the horizon of the merged solution has the topology $\left.S^{2} \times S^{D-4}\right]$

Consider now the Kerr-NUT-dS solution in D-dimensions [16], in units of $L=1$,

$$
\begin{aligned}
d s^{2}= & \rho^{2}\left(\frac{d r^{2}}{\Delta_{r}}+\frac{d u^{2}}{\Delta_{u}}\right)-\frac{\Delta_{r}}{\rho^{2} \Xi^{2}}\left(\left(1+a^{2} u^{2}\right) d t-a\left(1-u^{2}\right) d \phi\right)^{2} \\
& +\frac{\Delta_{u}}{\rho^{2} \Xi^{2}}\left(\left(r^{2}+a^{2}\right) d \phi-a\left(1-r^{2}\right) d t\right)^{2}+r^{2} u^{2} d \Omega_{(D-4)}^{2}
\end{aligned}
$$

with $\Delta_{r}$ and $\Xi$ as in (2.3), (2.5), and

$$
\rho^{2}=r^{2}+a^{2} u^{2}, \quad \Delta_{u}=\left(1-u^{2}\right)\left(1+a^{2} u^{2}\right)+\frac{2 N}{u^{D-5}} .
$$

Our notation and choice of parametrization is different than in [16], but more adequate for our purposes. If we set the NUT parameter $4=0$ and redefine $u=\cos \theta$ we recover

\footnotetext{
${ }^{3}$ Away from the critical solution, this could be a non-trivial bundle. This will not be important in our analysis.

${ }^{4}$ In the four-dimensional solution, the usual NUT parameter is not exactly the same as $N$ here. The relation is nevertheless easily found.
} 
(2.1). Note that having $N \neq 0$ in $D \geq 6$ prevents $u$ from reaching zero, since the last term in $\Delta_{u}$ would blow up.

We are interested in solutions with a single temperature, so we take the same limit as in appendix A to obtain a generalization of (2.13),

$$
\begin{aligned}
d s^{2}= & C \rho_{0}^{2}\left(-\sin ^{2} \chi d \tilde{t}^{2}+d \chi^{2}\right)+\frac{\rho_{0}^{2}}{\Delta_{u}} d u^{2} \\
& +\frac{\Delta_{u}}{\rho_{0}^{2} \Xi^{2}}\left(\left(r_{0}^{2}+a^{2}\right) d \tilde{\phi}-2 C r_{0} a \Xi \cos \chi d \tilde{t}\right)^{2}+r_{0}^{2} u^{2} d \Omega_{(D-4)}^{2}
\end{aligned}
$$

where

$$
\rho_{0}^{2}=r_{0}^{2}+a^{2} u^{2}
$$

and $r_{0}, C$ are the same functions of $a^{2}$ as in sec. 2.2. The only non-trivial dependence of the metric is on $u$.

There are two parameters, $a$ and $N$. In sec. 2.2 we set $N=0$ and $0 \leq a^{2} \leq \infty$, with the critical phase being reached as $a \rightarrow \infty$. Here we extend the solutions into a new parameter range by considering negative values of $a^{2}$, specifically

$$
-\infty \leq a^{2}<-1
$$

as covered when $r_{0}$ varies in 5

$$
\frac{D-5}{D-1}<r_{0}^{2} \leq \frac{D-5}{D-3}
$$

Note that this implies $0<r_{0}^{2}<1$, and also $M_{0}<0$. It is convenient to define

$$
\alpha^{2}=-a^{2}, \quad \hat{C}=C a^{2}
$$

so that $\alpha^{2}, \hat{C}>0$, and

$$
\begin{aligned}
& \Sigma(u)=\frac{\rho_{0}^{2}}{a^{2}}=u^{2}-\frac{r_{0}^{2}}{\alpha^{2}}, \\
& \Upsilon(u)=\frac{\Delta_{u}}{a^{2}}=\left(1-u^{2}\right)\left(u^{2}-\alpha^{-2}\right)+\frac{2 \hat{N}}{u^{D-5}},
\end{aligned}
$$

where we have conveniently absorbed a power of $\alpha$ in $\hat{N}$. The metric reads 6

$$
\begin{aligned}
d s^{2}= & \hat{C} \Sigma\left(-\sin ^{2} \chi d \tilde{t}^{2}+d \chi^{2}\right)+\frac{\Sigma}{\Upsilon} d u^{2} \\
& +\frac{\Upsilon}{\Sigma \Xi^{2}}\left(\left(\alpha^{2}-r_{0}^{2}\right) d \tilde{\phi}+2 i \hat{C} r_{0} \alpha \Xi \cos \chi d \tilde{t}\right)^{2}+r_{0}^{2} u^{2} d \Omega_{(D-4)}^{2}
\end{aligned}
$$

\footnotetext{
${ }^{5}$ This range of $a^{2}$ is also obtained with $1<r_{0}^{2} \leq \infty$, but one can see this is not adequate for obeying the required behavior.

${ }^{6}$ Refs. [17, 18, give the Euclidean version of this solution in a different parametrization that is more elegant but less appropriate for our purposes.
} 
This is a complex metric, with imaginary $g_{\tilde{t} \tilde{\phi}}$, since we are taking the rotation parameter $a$ into imaginary values. We will return to this issue below.

We take $u$ to vary in an interval for which $\Sigma$ and $\Upsilon$ are both non-negative. The zeroes of $\Upsilon$, which limit this interval, depend on the NUT parameter $\hat{N}$, which so far has been free. When $\hat{N}=0$, the range in which $\Upsilon$ is positive is

$$
\alpha^{-1} \leq u \leq 1 \quad(\hat{N}=0)
$$

(we need only consider $u>0$ ). Since for $\alpha<\infty, u$ is never zero, we fulfill one of the topological requirements on the merged solution: the $S^{D-4}$ never shrinks to zero size. However, since $\alpha^{-1}>r_{0} / \alpha$, we have that $\Sigma(u)$, although positive, is never zero and therefore the Lorentzian- $S_{\tilde{t}, \chi}^{2}$ is not contractible. This can be remedied by turning on the parameter $\hat{N}$ and tuning it so that the smallest of the two relevant roots of $\Upsilon$ moves to the value $r_{0} / \alpha$; the other will be $u_{1}>1$. Then we take

$$
\frac{r_{0}}{\alpha} \leq u \leq u_{1}
$$

Note that since now $\Sigma$ and $\Upsilon$ both have a simple zero at $u=r_{0} / \alpha$, the $\tilde{\phi}$ circle has finite size there.

While it is easy to solve for the required value of $\hat{N}$ as a function of $\alpha$, we are mostly interested in the regime where $\alpha$ is very large, in which we approach the critical solution. Then one finds

$$
\hat{N} \approx \frac{1}{D-3}\left(\frac{D-5}{D-3}\right)^{\frac{D-5}{2}} \frac{1}{\alpha^{D-3}}, \quad \frac{r_{0}}{\alpha} \approx \sqrt{\frac{D-5}{D-3}} \frac{1}{\alpha}, \quad u_{1} \approx 1+\hat{N} .
$$

Note that $\hat{N} \rightarrow 0$ as $\alpha \rightarrow \infty$.

With these parameter choices, we obtain a solution in which

- as $\alpha \rightarrow \infty$ the metric becomes that of the critical solution (2.21) (with $u=\cos \theta$ ),

- there is a contractible Lorentzian- $S^{2}$ and a non-contractible $S^{D-4}$.

- the constant- $\tilde{t}$ sections of the horizon, where $\sin \chi=0$, have topology $S^{2} \times S^{D-4}$. The $S^{2}$ is made of the two disks at $\chi=0, \pi$, joined along $u=r_{0} / \alpha$.

Therefore, this one-parameter family of solutions satisfies all the properties that we required at the beginning of the section.

Unfortunately, these geometries have two significant shortcomings. First, they are complex, and then it is unclear whether it is sensible to talk about a horizon. Second, and perhaps worse, when the Lorentzian- $S_{\tilde{t}, \chi}^{2}$ shrinks to zero at $u=r_{0} / \alpha$, it does not 
do so smoothly. Near this point, the $(u, \tilde{t}, \chi)$ part of the geometry behaves (up to constant factors) as

$$
\left(u-r_{0} / \alpha\right)\left(-\sin ^{2} \chi d \widetilde{t}^{2}+d \chi^{2}\right)+d u^{2}
$$

which is singular at $u=r_{0} / \alpha \rrbracket$

The problem of the Lorentzian metric being complex can be remedied by going to the Euclidean section. However, the trouble then comes back in that it does not seem possible to have a regular, real Euclidean section for the solution before the merger. It seems we cannot have the real transition both ways.

We have not found any other way of obeying the topology requirements of the merged solution using the family of metrics (3.1) than with these parameter choices. It is unclear whether there may be a more general solution that is better behaved.

\section{Critical geometries for other topology-changing transitions}

In sec. 2 we have presented an exact instance of an intersection of horizons that gives a satisfactory account of all the aspects of the pre-merger transition conforming to the analysis of [7]. Thus it seems justified to look for local models for the critical geometries in other horizon-merger transitions that are expected to occur for higher-dimensional black holes. We will see that there exist self-similar cone geometries with the adequate properties for all these transitions.

Black ring pinch. The simplest new critical geometry that we describe corresponds to the transition between a black ring with horizon topology $S^{1} \times S^{D-3}$ and a black hole with horizon topology $S^{D-2}$ in $D \geq 6$. In the black hole phase, the horizon geometry develops a pinch along the rotation axis, which grows until it pinches off in the critical solution. Coming from the black ring side, the ring becomes fatter until its central hole closes up. The pinch-off occurs on the rotation axis, so we can expect that asymptotically close to this point the rotation is negligible. Thus, the self-similar geometry around this point will be locally a static cone.

In the black ring phase the Euclidean time circle fibers over disks $D_{2}$ that fill the ring's hole, to form a $S^{3}$. In addition, on the horizon we can find spheres $S^{D-4}$ that shrink to zero size at the inner rim of the ring. Instead, in the black hole phase these $S^{D-4}$ do not shrink anywhere in the region close to the axis, while the previously described $S^{3}$ does shrink to zero there. Hence, we have an instance of a conifold-type

\footnotetext{
${ }^{7}$ The fact that these solutions have $M<0$ might be behind this.
} 


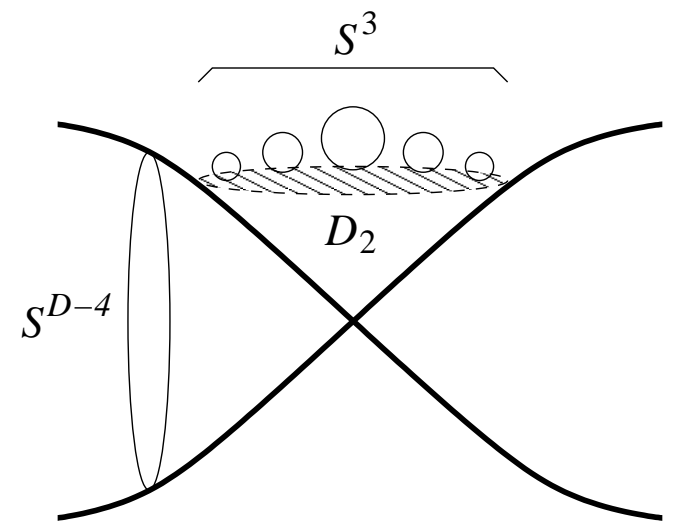

Figure 6: Critical geometry at the 'black ring pinch' transition between a black ring and a topologically spherical black hole. Relative to fig. 1, the main difference is that the dashed segment is replaced by a disk $D_{2}$. In general, for a $p$-sphere pinch, the disk $D_{2}$ is replaced by a ball $B_{p+1}$, and the $S^{3}$ by a $S^{p+2}$ ( $p=0$ is the case in fig. 11).

transition, with the critical geometry being a cone over $S^{3} \times S^{D-4}$,

$$
d s^{2}=d z^{2}+\frac{z^{2}}{D-2}\left(2 d \Omega_{(3)}^{2}+(D-5) d \Omega_{(D-4)}^{2}\right)
$$

(see fig. 6, this can be obtained by rotating the critical solution of fig. 1 around a vertical axis.). The Lorentzian version of the geometry is

$$
d s^{2}=d z^{2}+\frac{2 z^{2}}{D-2}\left(-\cos ^{2} \chi d t^{2}+d \chi^{2}+\sin ^{2} \chi d \phi^{2}+\frac{D-5}{2} d \Omega_{(D-4)}^{2}\right)
$$

with the horizon being at $\chi=\pi / 2$.

Note that this conifold transition can not occur for five-dimensional black rings since the required cone does not exist.

$p$-sphere pinch. Refs. [1, 13] generalized black rings with horizon $S^{1} \times S^{D-3}$ to solutions with horizon $S^{p} \times S^{D-p-2}$, with odd $p$, where the $S^{p}$ is a contractible cycle we refer to it as an 'odd-sphere blackfold'. Like in a black ring, the $S^{p}$ has to rotate in order to maintain its equilibrium at a given radius. The approximate method used in [1, 13] is only valid as long as the blackfold is thin, i.e., the $S^{p}$ is much larger than the $S^{D-p-2}$, but it is natural to expect that when the angular momentum decreases, the sphere $S^{p}$ shrinks until its inner hole closes up, like in the example of the black ring. At that point the configuration makes a transition to a topologically-spherical black hole.

In this case the inner hole is a ball $B_{p+1}$ over which the Euclidean time circle fibers, shrinking to zero at the boundary of the ball: this is a $S^{p+2}$. The size of this sphere 
remains finite in the odd-sphere-blackfold phase, and shrinks to zero at the axis in the topologically-spherical black hole phase. On the other hand the horizon has a $S^{D-p-3}$ that shrinks to zero size in the odd-sphere blackfold phase, and remains finite (near the axis) in the black hole phase. Thus we have another conifold transition, this time with a critical geometry

$$
d s^{2}=d z^{2}+\frac{p+1}{D-2} z^{2}\left(d \Omega_{(p+2)}^{2}+\frac{D-p-4}{p+1} d \Omega_{(D-p-3)}^{2}\right),
$$

and in the Lorentzian version

$$
d s^{2}=d z^{2}+\frac{p+1}{D-2} z^{2}\left(-\cos ^{2} \chi d t^{2}+d \chi^{2}+\sin ^{2} \chi d \Omega_{(p)}^{2}+\frac{D-p-4}{p+1} d \Omega_{(D-p-3)}^{2}\right)
$$

with $0 \leq \chi \leq \pi / 2$ and horizon at $\chi=\pi / 2$.

Note that the boundary of $B_{p+1}$ is connected when $p>0$ so, unlike in sec. 2 (which was locally the case $p=0$ smeared along a circle), in these cases the merger necessarily involves only one horizon.

In the odd-sphere blackfold, when the angular momenta are not all equal, the $S^{p}$ is not geometrically round. One may reduce some of the angular momenta while the others are kept fixed. In this case we expect a transition to an ultraspinning topologically spherical black hole, controlled by a local geometry of the type above with a collapsing sphere of lower dimensionality.

Circular pinch. Finally, we describe another type of critical pinch-off geometry that is expected to occur in the phase space of higher-dimensional rotating black holes. This is the case of a circular pinch at finite radius on the plane of rotation. This mediates the transition between, for instance, a black Saturn in $D \geq 6$ and a topologically spherical black hole with a circular pinch. The same local critical geometry also appears when two black rings in $D \geq 6$ merge.

The two black objects that merge are rotating, but as they approach we can go to a reference frame that is asymptotically corotating with the two horizons, and in this frame the geometry near the pinch-off point looks again static. This is then locally like in fig. 1, spread over the direction of the circle where the horizons touch. The local model for the critical geometry is the same as (2.22). Note again that this cannot occur in five dimensions.

It is actually possible to consider also intersecting geometries in which the two black objects have different temperatures, as in (2.10), where the horizons touch on a cone but cannot merge. What does not seem possible is to have the two horizons touch over a cone if they have relative non-zero velocity. Presumably, an attempt to force two such horizons to touch gives a stronger singularity. 
Given these examples, it is straightforward to extend them to cones for many other transitions that are expected to occur for higher-dimensional black holes.

\section{Concluding remarks}

The main features of the phase space of neutral, asymptotically flat, higher-dimensional black holes are controlled by solutions in three different regions:

(i) Large angular momenta.

(ii) Bifurcations in phase space.

(iii) Topology-changing transitions.

The regime (i) is captured by the blackfold effective theory [1, 2]. Regions (ii) are controlled by zero-mode perturbations of black holes that give rise to bifurcations into new families of solutions. The initial conjectures about these points [12, 10] have been confirmed and extended in [19]. In this paper we have begun to explore regions (iii) in $D \geq 6$ and provided local models for the critical geometries that effect the topology change.

We have presented an example where we can study in a detailed exact manner the geometry in a topology-changing transition, at least in one of the sides of the transition and at the critical point itself. The critical geometry conforms precisely to the predictions of [7]. It seems valuable to have an exact and simple analytic model of one such transition from which one can extract further details. In particular, a more detailed study of how the conical geometries, including the large class of examples in appendix B, are resolved away from the critical point is probably of interest.

We have also seen that self-similar cone geometries occur when two horizons intersect but cannot merge since their temperatures are unequal. In this case the cone is over a warped product, not a direct one, and the arguments of [7] would not apply. Nevertheless, the extension we have found is a natural one: the direct-product Nariai solution at the base of the cone is simply replaced by the more general SchwarzschilddeSitter solution. This allows to study intersecting horizons in more generality.

Topology-changing transitions among five-dimensional asymptotically flat rotating black holes do not fall within the class studied in this paper. Indeed, the phase diagram of five-dimensional black holes, say with a single spin, already reveals that the transitions are controlled by a different class of critical geometry, not of the conifold type. The same is true of transitions that are effectively in that same class by having 
some directions smeared along the intersection (e.g., black Saturn or black di-ring mergers), and in general those that involve a collapsing $S^{1}$. The study of these transitions deserves a separate investigation.

\section{Acknowledgments}

Work supported by MEC FPA2010-20807-C02-02, AGAUR 2009-SGR-168 and CPAN CSD2007-00042 Consolider-Ingenio 2010. We acknowledge hospitality and a highly stimulating environment at the Centro de Ciencias de Benasque Pedro Pascual during the Strings Workshop and the Gravity Workshop in July 2011, and in particular we are grateful to the participants for very useful discussions with many of them.

\section{A Equal-temperature limit}

In this appendix we define a limit of the Kerr-deSitter solutions (2.1) and the KerrNUT-deSitter solutions (3.1) that generalizes the well-known Nariai limit of the SchwdS solutions. We use units where the cosmological radius is $L=1$.

In order for the two horizons of (2.1) to have equal surface gravities, or equal temperatures, the value of the radial coordinate $r$ must be the same for both, i.e., $\Delta_{r}$ must have a double root $r=r_{0}$,

$$
\Delta_{r}\left(r_{0}\right)=\partial_{r} \Delta_{r}\left(r_{0}\right)=0
$$

These two conditions determine the values of $M$ and $a$ as functions of $r_{0}$,

$$
M_{0}=r_{0}^{D-3} \frac{\left(1-r_{0}^{2}\right)^{2}}{(D-3) r_{0}^{2}-(D-5)}, \quad a^{2}=r_{0}^{2} \frac{D-3-(D-1) r_{0}^{2}}{(D-3) r_{0}^{2}-(D-5)} .
$$

Although their radial coordinates coincide, the proper radial distance between the two horizons goes to a finite non-zero limit. We can pry open the space between them by first introducing a small parameter $\varepsilon$ that takes us slightly away from the limit, with a new 'radial' coordinate $\chi$,

$$
r=r_{0}(1-\varepsilon \cos \chi)
$$

and

$$
M=M_{0}\left(1-\frac{r_{0}^{2}}{C\left(r_{0}^{2}+a^{2}\right)\left(1-r_{0}^{2}\right)} \varepsilon^{2}\right),
$$

where $C$ is as in (2.17). Then redefine appropriately the Killing coordinates $t$ and $\phi$ to

$$
t=C \frac{r_{0}^{2}+a^{2}}{r_{0}} \frac{\tilde{t}}{\varepsilon}, \quad \phi=\tilde{\phi}+C \frac{a\left(1-r_{0}^{2}\right)}{r_{0}} \frac{\tilde{t}}{\varepsilon} .
$$

\footnotetext{
${ }^{8}$ Ref. [20] describes essentially the same limit, but in a different parametrization.
} 
Finally, take the limit $\varepsilon \rightarrow 0$ to find the finite metric (2.13).

The limit fixes one of the two dimensionless free parameters of the original solution, leaving $r_{0}$, or $a$, as the only parameter. In this paper we are interested in approaching the solutions that satisfy (2.7), (2.8). In the one-parameter family, this corresponds to

$$
r_{0}^{2} \rightarrow \frac{D-5}{D-3}
$$

Indeed in this limit

$$
a \rightarrow \infty, \quad M_{0} \rightarrow \infty
$$

while

$$
\frac{M_{0}}{a^{2}} \rightarrow \frac{1}{D-5}\left(\frac{D-5}{D-3}\right)^{\frac{D-3}{2}}
$$

remains finite. Also, in this limit $C a^{2} \rightarrow 1 /(D-3)$.

\section{B General black hole-deSitter intersections}

Here we extend the analysis of sec. 2 to the situation where the black hole rotates in an arbitrary number of planes. The starting solution is the general Kerr-deSitter metric as given in ref. [21], whose presentation we follow closely.

In order to have a unified description for even and odd $D$, we introduce

$$
\epsilon=(D-1) \bmod 2 \text {. }
$$

The spacetime dimension is then $D=2 n+\epsilon+1$, where $n$ is the number of orthogonal rotation planes. On each of these, we choose angles $\phi_{i}$ with period $2 \pi$. We also introduce an overall radial coordinate $r$ and $n+\epsilon$ direction cosines $\mu_{i}$ satisfying

$$
\sum_{i=1}^{n+\epsilon} \mu_{i}^{2}=1
$$

with $0 \leq \mu_{i} \leq 1, i=1, \ldots, n$. In even $D$ the $\mu_{n+1}$ has range $-1 \leq \mu_{n+1} \leq 1$.

The solution is characterized by a mass parameter $M$ and $n$ rotation parameters $a_{i}$, and its metric is

$$
\begin{aligned}
d s^{2}= & -W\left(1-\frac{r^{2}}{L^{2}}\right) d t^{2}+\frac{2 M}{U}\left(W d t-\sum_{i=1}^{n} \frac{a_{i} \mu_{i}^{2}}{1+\frac{a_{i}^{2}}{L^{2}}} d \phi_{i}\right)^{2}+\sum_{i=1}^{n} \frac{r^{2}+a_{i}^{2}}{1+\frac{a_{i}^{2}}{L^{2}}} \mu_{i}^{2} d \phi_{i}^{2} \\
& +\frac{U}{V-2 M} d r^{2}+\sum_{i=1}^{n+\epsilon} \frac{r^{2}+a_{i}^{2}}{1+\frac{a_{i}^{2}}{L^{2}}} d \mu_{i}^{2}+\frac{1}{W\left(L^{2}-r^{2}\right)}\left(\sum_{i=1}^{n+\epsilon} \frac{\left(r^{2}+a_{i}^{2}\right) \mu_{i} d \mu_{i}}{1+\frac{a_{i}^{2}}{L^{2}}}\right)^{2}
\end{aligned}
$$


where

$$
\begin{gathered}
U=r^{\epsilon} \sum_{i=1}^{n+\epsilon} \frac{\mu_{i}^{2}}{r^{2}+a_{i}^{2}} \prod_{l=1}^{n}\left(r^{2}+a_{l}^{2}\right), \\
V=r^{\epsilon-2}\left(1-\frac{r^{2}}{L^{2}}\right) \prod_{i=1}^{n}\left(r^{2}+a_{i}^{2}\right),
\end{gathered}
$$

and

$$
W=\sum_{i=1}^{n+\epsilon} \frac{\mu_{i}^{2}}{1+\frac{a_{i}^{2}}{L^{2}}} .
$$

We take the limit in which we send a number $s$ of rotation parameters to infinity, all at the same rate. For now, the remaining $n-s$ ones are set to zero, so

$$
\begin{array}{rlrl}
a_{j} \rightarrow \infty, & j & =1, \ldots, s, \\
a_{k} & =0, & k & =s+1, \ldots, n,
\end{array}
$$

with

$$
2 s \leq D-4 .
$$

At the same time we send $M \rightarrow \infty$ in such a way that

$$
\mu=\frac{2 M}{\prod_{j=1}^{s} a_{j}^{2}}
$$

remains finite (compare to appendix A of [12]).

We introduce an angular variable $\theta$ such that

$$
\sum_{j=1}^{s} \mu_{j}^{2}=\sin ^{2} \theta, \quad \sum_{k=s+1}^{n+\epsilon} \mu_{k}^{2}=\cos ^{2} \theta
$$

with range $0 \leq \theta \leq \pi / 2$. With this variable, the metric on the unit $S^{D-2}$ is written as

$$
\sum_{i=1}^{n+\epsilon} d \mu_{i}^{2}+\sum_{i=1}^{n} \mu_{i}^{2} d \phi_{i}^{2}=d \Omega_{(D-2)}^{2}=d \theta^{2}+\sin ^{2} \theta d \Omega_{(2 s-1)}^{2}+\cos ^{2} \theta d \Omega_{(D-2 s-2)}^{2} .
$$

After some labor one finds the limiting geometry

$$
d s^{2}=L^{2}\left(d \theta^{2}+\sin ^{2} \theta d \Omega_{(2 s-1)}^{2}\right)+\cos ^{2} \theta\left(-f(r) d t^{2}+\frac{d r^{2}}{f(r)}+r^{2} d \Omega_{(D-2 s-2)}^{2}\right)
$$

with

$$
f(r)=1-\frac{\mu}{r^{D-2 s-3}}-\frac{r^{2}}{L^{2}} .
$$

Near $\theta=\pi / 2$ this has the form of a cone over $\mathrm{Schw}-\mathrm{dS}_{D-2 s}$, spread over a sphere $S^{2 s-1}$. When $s=1$ we recover (2.9). The restriction (B.8) on the number $s$ of 'ultraspins' guarantees that the $\mathrm{Schw}-\mathrm{dS}_{D-2 s}$ factor in the limit geometry is at least four-dimensional. 
It is easy to generalize the limit to

$$
\begin{aligned}
a_{j} \rightarrow \infty, & j=1, \ldots, s, \\
a_{k} \text { finite, } & k=s+1, \ldots, n,
\end{aligned}
$$

again with finite $\mu$ in $(\underline{B .9})$ to obtain the geometry

$$
d s^{2}=L^{2}\left(d \theta^{2}+\sin ^{2} \theta d \Omega_{(2 s-1)}^{2}\right)+\cos ^{2} \theta d s^{2}\left(\operatorname{Kerr}-\mathrm{dS}{ }_{(D-2 s)}\right)
$$

where Kerr-dS $\mathrm{S}_{(D-2 s)}$ has the finite $a_{k}$ as rotation parameters. The Euclidean version of the latter solution is known to contain many interesting Einstein metrics, in addition to products $S^{2} \times S^{D-2 s-2}$, such as Page's metric for the non-trivial $S^{2}$ bundle over $S^{2}$ [22] and higher-dimensional generalizations thereof (see e.g., [17] and references to it). Our construction results in cones over all these spaces. It may be interesting to study them in more detail.

\section{References}

[1] R. Emparan, T. Harmark, V. Niarchos and N. A. Obers, "Worldvolume Effective Theory for Higher-Dimensional Black Holes (Blackfolds)," Phys. Rev. Lett. 102, 191301 (2009) arXiv:0902.0427 [hep-th]].

[2] R. Emparan, T. Harmark, V. Niarchos and N. A. Obers, "Essentials of Blackfold Dynamics," JHEP 1003 (2010) 063 [arXiv:0910.1601 [hep-th]].

[3] B. Kol, "The Phase transition between caged black holes and black strings: A Review," Phys. Rept. 422 (2006) 119-165. hep-th/0411240].

T. Harmark, N. A. Obers, "Phases of Kaluza-Klein black holes: A Brief review," hep-th/0503020].

G. T. Horowitz, T. Wiseman, "General black holes in Kaluza-Klein theory," arXiv:1107.5563 [gr-qc]].

[4] S. S. Gubser, "On nonuniform black branes," Class. Quant. Grav. 19 (2002) 4825-4844. hep-th/0110193.

T. Wiseman, "Static axisymmetric vacuum solutions and nonuniform black strings," Class. Quant. Grav. 20 (2003) 1137-1176. hep-th/0209051.

B. Kleihaus, J. Kunz, E. Radu, "New nonuniform black string solutions," JHEP 0606 (2006) 016. hep-th/0603119.

E. Sorkin, "Non-uniform black strings in various dimensions," Phys. Rev. D74 (2006) 104027. gr-qc/0608115]. 
[5] T. Harmark, N. A. Obers, "Black holes on cylinders," JHEP 0205 (2002) 032. [hep-th/0204047].

T. Wiseman, "From black strings to black holes," Class. Quant. Grav. 20 (2003) 1177-1186. hep-th/0211028.

B. Kol, E. Sorkin, T. Piran, "Caged black holes: Black holes in compactified space-times. 1. Theory," Phys. Rev. D69 (2004) 064031. hep-th/0309190.

E. Sorkin, B. Kol, T. Piran, "Caged black holes: Black holes in compactified space-times. 2. 5-d numerical implementation," Phys. Rev. D69 (2004) 064032. hep-th/0310096.

T. Harmark, N. A. Obers, "Phase structure of black holes and strings on cylinders," Nucl. Phys. B684 (2004) 183-208. [hep-th/0309230].

H. Kudoh, T. Wiseman, "Properties of Kaluza-Klein black holes," Prog. Theor. Phys. 111 (2004) 475-507. hep-th/0310104.

H. Kudoh, T. Wiseman, "Connecting black holes and black strings," Phys. Rev. Lett. 94 (2005) 161102. hep-th/0409111.

[6] M. Headrick, S. Kitchen, T. Wiseman, "A New approach to static numerical relativity, and its application to Kaluza-Klein black holes," Class. Quant. Grav. 27 (2010) 035002. arXiv:0905.1822 [gr-qc]].

[7] B. Kol " Topology change in General Relativity, and the black-hole black-string transition," JHEP 10 (2005) 049 [arXiv:hep-th/0206220].

[8] B. Kol, T. Wiseman " Evidence that highly non-uniform black strings have a conical waist," Class. Quant. Grav. 20 (2003) 3493 [arXiv:hep-th/0304070v2].

[9] V. Asnin, B. Kol, M. Smolkin, "Analytic evidence for continuous self similarity of the critical merger solution," Class. Quant. Grav. 23 (2006) 6805-6827. [hep-th/0607129.

[10] R. Emparan, T. Harmark, V. Niarchos, N. A. Obers and M. J. Rodríguez, "The Phase Structure of Higher-Dimensional Black Rings and Black Holes," JHEP 0710 (2007) 110 arXiv:0708.2181 [hep-th]].

[11] R. Emparan, P. Figueras, "Multi-black rings and the phase diagram of higherdimensional black holes," JHEP 1011 (2010) 022. [arXiv:1008.3243 [hep-th]].

[12] R. Emparan, R. C. Myers, "Instability of ultra-spinning black holes," JHEP 0309 (2003) 025. arXiv:hep-th/0308056 [hep-th]]. 
[13] R. Emparan, T. Harmark, V. Niarchos and N. A. Obers, "New Horizons for Black Holes and Branes," JHEP 1004 (2010) 046. arXiv:0912.2352 [hep-th]].

[14] L. Lehner, F. Pretorius, "Black Strings, Low Viscosity Fluids, and Violation of Cosmic Censorship," Phys. Rev. Lett. 105 (2010) 101102. arXiv:1006.5960 [hepth]].

[15] S. W. Hawking, C. J. Hunter, M. Taylor, "Rotation and the AdS / CFT correspondence," Phys. Rev. D59 (1999) 064005. hep-th/9811056.

[16] D. Klemm, "Rotating black branes wrapped on Einstein spaces," JHEP 9811 (1998) 019. hep-th/9811126.

[17] H. Lu, D. N. Page, C. N. Pope, "New inhomogeneous Einstein metrics on sphere bundles over Einstein-Kahler manifolds," Phys. Lett. B593 (2004) 218-226. [hep-th/0403079.

[18] R. B. Mann, C. Stelea, "New multiply nutty spacetimes," Phys. Lett. B634 (2006) 448-455. hep-th/0508203.

[19] O. J. C. Dias, P. Figueras, R. Monteiro, J. E. Santos, R. Emparan, "Instability and new phases of higher-dimensional rotating black holes," Phys. Rev. D80 (2009) 111701. arXiv:0907.2248 [hep-th]].

O. J. C. Dias, P. Figueras, R. Monteiro, J. E. Santos, "Ultraspinning instability of rotating black holes," Phys. Rev. D82 (2010) 104025. [arXiv:1006.1904 [hep-th]].

M. Durkee, H. S. Reall, "Perturbations of near-horizon geometries and instabilities of Myers-Perry black holes," Phys. Rev. D83 (2011) 104044. arXiv:1012.4805 [hep-th]].

O. J. C. Dias, R. Monteiro, J. E. Santos, "Ultraspinning instability: the missing link," JHEP 1108 (2011) 139. arXiv:1106.4554 [hep-th]].

[20] Z. W. Chong, G. W. Gibbons, H. Lu, C. N. Pope, "Separability and killing tensors in Kerr-Taub-NUT-de sitter metrics in higher dimensions," Phys. Lett. B609 (2005) 124-132. hep-th/0405061.

[21] G. W. Gibbons, H. Lu, D. N. Page, C. N. Pope, "Rotating black holes in higher dimensions with a cosmological constant," Phys. Rev. Lett. 93 (2004) 171102. hep-th/0409155.

[22] D. N. Page, "A Compact Rotating Gravitational Instanton," Phys. Lett. B79 (1978) 235. 